\title{
Qualidade de vida relacionada à saúde de idosos em tratamento quimioterápico
}

\author{
Health-related quality of life of elderly people undergoing chemotherapy
}

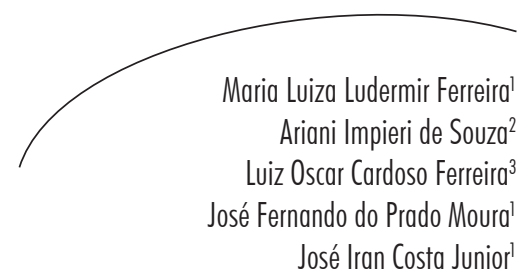

Resumo

Objetivo: Avaliar os efeitos da quimioterapia antineoplásica na qualidade de vida relacionada à saúde (QVRS) de idosos. Método: Foi analisada uma série de casos de idosos submetidos a quimioterapia antineoplásica, selecionados por amostra não probabilística do tipo tempo-local no período de agosto a dezembro de 2012. Foram incluídos idosos em tratamento quimioterápico e excluídos aqueles com indicação de radioterapia concomitante à quimioterapia. Para avaliação da QVRS, foi utilizado o European Organization for Research and Treatment of Cancer Quality of Life Questionnaire "core" 30 item (EORTC-QLQ-C30), aplicado antes e cerca de dois meses após o início da quimioterapia. A qualidade de vida foi avaliada por meio da comparação das médias dos escores antes e depois da quimioterapia, por meio do teste $t$ Student. Resultados: Dos 31 pacientes observados, 58,1\% eram do sexo feminino. As neoplasias mais frequentes foram: mama (32,3\%), pulmão $(22,6 \%)$ e próstata $(16,1 \%)$, sendo que $51,6 \%$ tinham o estadiamento clínico TNM IV. Para os 28 pacientes avaliados na segunda entrevista, o domínio "desempenho físico" teve variação da média "antes" e "depois" estatisticamente significante ( $\mathrm{p}=0,008)$, enquanto o domínio "estado de saúde geral/ QV" teve média na primeira entrevista de 69,3 pontos e, após dois meses, 64,3 pontos sem diferença estatística $(\mathrm{p}=0,413)$. Quando se analisou a QVRS por tipo de tumor, houve piora significativa nas médias dos escores no domínio "estado de saúde geral/ QV” para os pacientes com neoplasia da próstata $(\mathrm{p}=0,042)$. Conclusão: A quimioterapia piorou o desempenho físico sem modificar o estado geral de saúde de idosos, exceto para os pacientes com neoplasia da próstata.

\footnotetext{
Instituto de Medicina Integral Prof. Fernando Figueira. Serviço de Oncologia Clínica. Recife, PE, Brasil.

2 Instituto de Medicina Integral Prof. Fernando Figueira. Programa de Pós-graduação em Saúde Maternoinfantil. Recife, PE, Brasil.

3 Universidade de Pernambuco. Programa de Pós-graduação de Enfermagem em Promoção da Saúde. Recife, PE, Brasil.
}

Palavras-chave: Idoso. Qualidade de Vida. Neoplasias. Quimioterapia. 


\section{Abstract}

Objective: To evaluate cancer chemotherapy effects on health related quality of life (HRQoL) of elderly. Method: A series of cases were analyzed on elderly undergoing anticancer chemotherapy selected by non-probability sample of the standard timeplace from August to December 2012. The study included individuals undergoing chemotherapy and excluded those with indication for radiotherapy with chemotherapy. To evaluate HRQoL, we applied the European Organization for Research and Treatment of Cancer Quality of Life Questionnaire "core" 30 item (EORTCQLQ-C30) before and two months after starting chemotherapy. The quality of life was assessed by comparing the mean scores before and after chemotherapy by Student $t$ test. Results: Of 31 patients observed, 58.1\% were female. The most common cancer types were breast $(32.3 \%)$, lung $(22.6 \%)$ and prostate $(16.1 \%)$, and $51.6 \%$ were TNM IV classification. Of the 28 patients evaluated in the second interview, the domain "physical functioning" mean was statistically significant between "before" and "after" $(\mathrm{p}=0.008)$ when evaluated all patients, while the domain "global health status/QoL" mean was 69.3 points in the first interview and 64.3 points after two months, with no statistical difference $(\mathrm{p}=0.413)$. When assessed by type of cancer, there was a worsening in mean scores in the domain "global health status/QoL", in patients with prostate cancer $(\mathrm{p}=0.042)$. Conclusion: Cancer chemotherapy worsened physical functioning without modifying the overall health status of the elderly, except for patients with prostate cancer.
Key words: Elderly. Quality of Life. Neoplasms. Drug Therapy.

\section{INTRODUÇÃO}

O envelhecimento populacional produziu importantes mudanças no perfil das enfermidades, e nas últimas décadas tem sido observado um aumento na magnitude das neoplasias malignas. ${ }^{1}$ Estima-se que 26 milhões de novos casos de câncer serão diagnosticados no mundo até $2030^{1}$ e que mais de $50 \%$ desses casos ocorrerão em idosos, que é também o grupo de maior taxa de mortalidade por essa doença. ${ }^{2}$

Dentre as formas de tratamento das neoplasias, a quimioterapia é a mais frequente, e até $70 \%$ dos tumores necessitarão de tratamento quimioterápico em algum momento do curso da doença. ${ }^{3} \mathrm{~A}$ avaliação da qualidade de vida (QV) de pacientes em tratamento quimioterápico possibilita melhor compreensão da intensidade e quantidade dos sintomas do paciente e da importância que isso tem na sua saúde, permitindo também melhorar o planejamento da

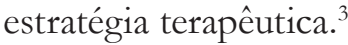

A QV é uma noção eminentemente humana, que tem sido correlacionada ao grau de satisfação encontrado na vida familiar, amorosa, social e ambiental, e à própria estética existencial. ${ }^{4} \mathrm{Na}$ área de saúde, o conceito mais utilizado tem sido o de "qualidade de vida relacionada à saúde" (QVRS) que carece de definição única. Diferente da QV, que é um conceito amplo, abrangendo diversos aspectos da vida, a QVRS refere-se ao valor que se possa atribuir à vida, decorrente de modificações que podem ocorrer por danos no estado funcional, percepções e fatores sociais, quando influenciados por doenças ou agravos, tratamentos e políticas de saúde. Parece estar mais diretamente associada às enfermidades e às intervenções em saúde. ${ }^{4}$

Para alguns pacientes idosos com câncer, a manutenção da QVRS tem sido o objetivo primordial do tratamento, sobrepondo-se inclusive ao aumento de sobrevida, já que essa população tem riscos adicionais de morte, além do câncer. Ademais, o tratamento pode 
acarretar mais efeitos colaterais em idosos do que em pacientes jovens, e essa toxicidade pode deteriorar a QVRS desses pacientes, colocando em discussão a melhor opção terapêutica., ${ }^{2,3,5}$

Ainda não existe uminstrumento padronizado para avaliar QVRS em pacientes idosos com câncer. O European Organization for Research and Treatment of Cancer Quality of Life Questionnaire "core" 30 item (EORTC QLQ-C30) é um questionário câncer-específico, multidimensional e aplicável a diferentes culturas. ${ }^{6}$ Já foi utilizado em diversos estudos clínicos com idosos, mesmo não tendo sido elaborado especificamente para esse grupo de pacientes. ${ }^{7-13}$ Sua adaptação para o Brasil foi realizada em um estudo que avaliou pacientes com neoplasia de pulmão. Ressalta-se que neste estudo a maioria dos pacientes era idosa. ${ }^{14}$

A identificação das particularidades dos idosos com câncer submetidos a quimioterapia antineoplásica faz-se necessária por meio de estudos que avaliem a qualidade de vida e, assim, melhorem a abordagem terapêutica desse subgrupo de pacientes. ${ }^{5,12}$ Objetivou-se, aqui, avaliar os efeitos da quimioterapia antineoplásica na qualidade de vida relacionada à saúde (QVRS) de idosos.

\section{METODOLOGIA}

Desenvolveu-se estudo longitudinal do tipo antes e depois, entre idosos submetidos a quimioterapia antineoplásica em um hospital que atende exclusivamente a pacientes do Sistema Único de Saúde (SUS) na cidade do Recife-PE. O referido hospital possui diferentes áreas de especialidade, dentre elas a oncologia clínica, para qual é referência no estado de Pernambuco e está cadastrado no Ministério da Saúde como um Centro de Assistência de Alta Complexidade em Oncologia (CACON). ${ }^{15}$

A amostra foi de conveniência no período de agosto a dezembro de 2012. Foram incluídos idosos (idade $\geq 60$ anos) com diagnóstico de neoplasia confirmado por histopatológico e que estivessem iniciando tratamento quimioterápico no ambulatório. Foram excluídos do estudo pacientes com indicação de radioterapia concomitante à quimioterapia. No período do estudo, o ambulatório da oncologia atendeu a 63 pacientes que preencheram os critérios de inclusão, dos quais foram recrutados 31 para a pesquisa, que estavam no ambulatório durante o período de trabalho dos entrevistadores.

As informações sociodemográficas e clínicas foram obtidas por meio de entrevistas registradas em um questionário elaborado para a presente pesquisa e mediante consulta ao prontuário. A qualidade de vida foi avaliada em dois momentos: antes do início do tratamento e após cerca de dois meses da inclusão no estudo, utilizando-se o questionário da EORTC- QLQ-C30, versão 3.0, validado para o português. ${ }^{14}$ Foi solicitada à instituição europeia autorização para sua aplicação no estudo.

O instrumento é composto por cinco escalas funcionais: função física, cognitiva, emocional, social e desempenho de papéis, uma escala de qualidade de vida e saúde global e, ainda, itens únicos sobre: fadiga, dor, náuseas, vômitos, dispneia, falta de apetite, insônia, constipação, diarreia e dificuldades financeiras. As respostas possíveis para as 28 perguntas iniciais são: "não", "pouco", "moderadamente" e "muito", pontuadas numa escala tipo Likert de "1 a 4". Para as outras duas questões finais, os valores variam de "1 a 7", sendo a pontuação "1" péssimo e a "7", ótimo. Dessa forma, o QLQ-C30 gera uma pontuação que pode somar de 0 a 100, de acordo com três fórmulas que constam no manual de escore da EORTC. Na escala funcional e saúde geral/QV, o zero denota o pior funcionamento e o 100 , o melhor; enquanto que na escala de sintomas ocorre o oposto, sendo o zero a ausência de sintomas e o 100 correspondendo ao máximo de sintomas presentes. ${ }^{6}$

As variáveis sociodemográficas utilizadas foram: idade, raça/cor, sexo, situação conjugal, escolaridade, situação de trabalho, renda familiar, procedência e religião. As variáveis clínicas foram: diagnóstico oncológico, Performance Status (ECOG-PS), escala utilizada para avaliar a situação clínica do paciente, ${ }^{16}$ localização e estadiamento do tumor (TNM), tipo de quimioterapia e comorbidades. 
Os dados foram processados e analisados no programa Epi- Info versão 3.5.3 e STATA versão 12.1SE. O perfil sociodemográfico e clínico foi apresentado por meio de medidas de frequência. O teste de homogeneidade marginal de StuartMaxwell foi utilizado para comparar o Perfomance Status antes e depois da quimioterapia. O efeito da quimioterapia na qualidade de vida foi avaliado por meio da comparação das médias dos escores antes e depois da quimioterapia, utilizando-se o teste $t$ Student. Em todas as análises, foi admitido o nível de significância de 5\%.

O estudo foi aprovado pelo Comitê de Ética em Pesquisa do Instituto de Medicina Integral Prof. Fernando Figueira, sob o número 3019/2012, e cumpre os princípios éticos contidos na Declaração de Helsinki, bem como a Resolução no 466/2012 do Conselho Nacional de Saúde. Todos os participantes assinaram o Termo de Consentimento Livre e Esclarecido antes de serem incluídos no estudo.

\section{RESULTADOS}

Durante o período do estudo, ocorreram dois óbitos e uma perda de seguimento por não comparecimento do paciente no período estabelecido para a segunda entrevista. Desse modo, dos 31 pacientes recrutados, 28 concluíram o estudo.

A média do intervalo de tempo entre a primeira e a segunda entrevista foi 62,5 dias ( $\mathrm{d} \mathrm{p} \pm 14,0)$, observando-se intervalo mínimo de 21 e máximo de 86 dias. A idade dos pacientes variou de 61 a 79 anos, obtendo-se média de 69,4 anos (dp $\pm 5,7)$.

Na tabela 1, estão descritas as características sociodemográficas dos 31 pacientes recrutados para o estudo. Havia $20,0 \%$ de analfabetos e $64,5 \%$ dos pacientes referiram ter cursado o ensino fundamental. Mais da metade $(80,6 \%)$ estavam aposentados ou de licença saúde. A renda familiar foi de até dois salários mínimos em $77,4 \%$ dos idosos do estudo. 
Tabela 1. Características sociodemográficas dos idosos em tratamento quimioterápico atendidos no Instituto de Medicina Integral Prof. Fernando Figueira. Recife-PE, 2012-2013.

\begin{tabular}{|c|c|c|}
\hline Características sociodemográficas & $\mathrm{n}=31$ & $\%$ \\
\hline \multicolumn{3}{|l|}{ Sexo } \\
\hline Feminino & 18 & 58,1 \\
\hline Masculino & 13 & 41,9 \\
\hline \multicolumn{3}{|l|}{ Raça/cor } \\
\hline Branca & 12 & 38,7 \\
\hline Parda/negra & 19 & 61,3 \\
\hline \multicolumn{3}{|l|}{ Situação conjugal } \\
\hline Sem parceiro & 11 & 35,5 \\
\hline Em união & 20 & 64,5 \\
\hline \multicolumn{3}{|l|}{ Escolaridade } \\
\hline Analfabeto & 6 & 19,4 \\
\hline Ensino fundamental & 20 & 64,5 \\
\hline Ensino médio ou mais & 5 & 16,1 \\
\hline \multicolumn{3}{|l|}{ Situação de trabalho } \\
\hline Empregado & 1 & 3,2 \\
\hline Desempregado & 3 & 9,7 \\
\hline Aposentado/licença saúde & 25 & 80,6 \\
\hline Do lar & 2 & 6,5 \\
\hline \multicolumn{3}{|l|}{ Religião } \\
\hline Católica & 24 & 77,4 \\
\hline Evangélica/outra & 7 & 22,6 \\
\hline \multicolumn{3}{|l|}{ Procedência } \\
\hline Recife e Região Metropolitana & 22 & 71,0 \\
\hline Interior & 9 & 29,0 \\
\hline \multicolumn{3}{|l|}{ Renda familiar (em salário mínimo*) } \\
\hline Até 2 & 24 & 77,4 \\
\hline$>2-\leq 4$ & 3 & 9,7 \\
\hline$>4$ & 4 & 12,9 \\
\hline
\end{tabular}

*Salário mínimo utilizado R\$622,00 referente ao ano 2012. 
Entre as comorbidades, a hipertensão arterial sistêmica (HAS) isolada ou associada ao diabete melito tipo 2 foi a mais relatada $(54,9 \%)$. Tumor de mama foi o diagnóstico mais frequente, com $32,3 \%$ (10/31), seguida pelo tumor de pulmão, em 22,6\% (7/31), e de próstata, 16,1\% (5/31). De acordo com os critérios do TNM, a doença tinha um estadiamento clínico II em 29,0\%, III em
19,3\% e IV em 51,6\% dos pacientes. Em 32,3\% dos idosos, os quimioterápicos mais utilizados foram adriamicina associada a ciclofosfamida, seguida de paclitaxel (AC-T) ou somente adriamicina associada a ciclofosfamida (AC). O paclitaxel combinado com a carboplatina foi utilizado em sete idosos (22,6\%), já o docetaxel, em cinco $(16,1 \%)$, segundo a tabela 2 .

Tabela 2. Características clínicas dos idosos em tratamento quimioterápico atendidos no Instituto de Medicina Integral Prof. Fernando Figueira. Recife-PE, 2012-2013.

\begin{tabular}{|c|c|c|}
\hline Características clínicas & $\mathrm{n}=31$ & $\%$ \\
\hline \multicolumn{3}{|l|}{ Comorbidade } \\
\hline HAS* & 13 & 42,0 \\
\hline $\mathrm{HAS}+\mathrm{DM} 2 * *$ & 4 & 12,9 \\
\hline DM2 & 2 & 6,4 \\
\hline Outros & 12 & 38,7 \\
\hline \multicolumn{3}{|l|}{ Diagnóstico oncológico } \\
\hline Mama & 10 & 32,3 \\
\hline Pulmão & 7 & 22,6 \\
\hline Próstata & 5 & 16,1 \\
\hline Outros & 9 & 29,0 \\
\hline \multicolumn{3}{|l|}{ Estadiamento $\mathrm{TNM}^{* * *}$} \\
\hline II & 9 & 29,0 \\
\hline III & 6 & 19,4 \\
\hline IV & 16 & 51,6 \\
\hline \multicolumn{3}{|l|}{ Quimioterápicos utilizados } \\
\hline $\mathrm{AC}-\mathrm{T} / \mathrm{AC}$ & 10 & 32,3 \\
\hline Paclitaxel + Carboplatina & 7 & 22,6 \\
\hline Docetaxel & 5 & 16,1 \\
\hline Gencitabina & 1 & 3,2 \\
\hline Outras combinações & 8 & 25,8 \\
\hline
\end{tabular}

*HAS= hipertensão arterial sistêmica; $* * \mathrm{DM} 2=$ diabete melito tipo $2 ; * * * \mathrm{TNM}=$ tumor, node e metastasis; $\$ AC-T/AC= adriamicina, ciclofosfamida- paclitaxel/adriamicina, ciclofosfamida. 
Entre os 28 idosos que voltaram para a segunda entrevista, a maioria $(63,3 \%)$ realizou o tratamento sem interrupção. Nos 11 (23,3\%) pacientes que suspenderam o tratamento, $\mathrm{O}$ principal motivo foi toxicidade medicamentosa. Para a comparação dos resultados do momento "antes" com o momento "depois", foram utilizados os 28 pacientes que concluíram a segunda entrevista do estudo.
Ao avaliar a pontuação na escala de Performance status (ECOG-PS) 85,7\% (24/28) dos pacientes iniciaram o estudo com EGOG-PS entre 0 e 1, e após o início do tratamento, 57,1\% (16/28) dos pacientes tinham essa mesma performance. Apenas três pacientes $(10,7 \%)$ melhoraram a pontuação na escala do ECOG-PS e 12 pioraram sua pontuação $(42,9 \%)$. Deste modo, o teste de homogeneidade marginal de Stuart-Maxwell resultou em um valor de $\mathrm{p}=0,114$ (tabela 3).

Tabela 3. Pontuação na escala de Performance Status (ECOG-PS) "antes" e "depois" do início do tratamento quimioterápico de idosos atendidos no Instituto de Medicina Integral Prof. Fernando Figueira. Recife-PE, 2012-2013.

\begin{tabular}{lllllcc}
\hline ECOG* antes & \multicolumn{2}{l}{ ECOG* depois } & & & \\
\hline & 0 & 1 & 2 & 3 & 4 & Total \\
0 & 5 & 2 & 1 & 1 & 1 & 10 \\
1 & 1 & 7 & 4 & 2 & 0 & 14 \\
2 & 0 & 1 & 1 & 0 & 1 & 3 \\
3 & 0 & 0 & 1 & 0 & 0 & 1 \\
4 & 0 & 0 & 0 & 0 & 0 & 0 \\
Total & 6 & 10 & 7 & 3 & 2 & $28^{* *}$ \\
\hline
\end{tabular}

*Teste de homogeneidade marginal de Stuart-Maxwell: $\mathrm{p}=0,114 ; * *$ três pacientes perderam seguimento.

Os escores de qualidade de vida avaliados com o EORTC QLQ-C30 estão descritos na tabela 4. O domínio "desempenho físico" teve média de 81,4 pontos na primeira entrevista, antes do início da quimioterapia e dois meses após essa média diminuiu para 64,7, evidenciando piora dessa função com diferença estatisticamente significativa $\quad(\mathrm{p}=0,008)$. Os domínios "desempenho funcional", "desempenho cognitivo" e "desempenho social" obtiveram diminuição dos escores e o "desempenho emocional" mostrou aumento entre a primeira e a segunda avaliação, mas todos sem significância estatística. Os domínios da escala de sintomas e itens únicos - dor, fadiga, náuseas e vômitos, dispneia, perda de apetite e diarreia - aumentaram a pontuação do escore (piora dos sintomas), enquanto os domínios "insônia", "constipação" e "dificuldades financeiras" diminuíram sua pontuação no escore (melhora dos sintomas), mas também sem diferença estatisticamente significativa entre os momentos "antes" e “depois". Em relação ao domínio "estado geral de saúde/QV", a média na primeira entrevista foi de 69,3 (dp $\pm 23,1)$ e após cerca de dois meses, 64,3 (dp $\pm 32,2)$, sem diferença estatística entre os escores $(\mathrm{p}=0,413)$. 
Tabela 4. Escores de qualidade de vida avaliados com o EORTC QLQ-C30 no momento "antes" e "depois" do tratamento quimioterápico de idosos atendidos no Instituto de Medicina Integral Prof. Fernando Figueira. Recife-PE, 2012-2013.

\begin{tabular}{lcccc}
\hline & $\begin{array}{c}\text { Antes } \\
\text { média (dp) }\end{array}$ & $\begin{array}{c}\text { Depois } \\
\text { média (dp) }\end{array}$ & $\begin{array}{c}\Delta \\
(\mathrm{D}-\mathrm{A})\end{array}$ & $\mathrm{p}^{*}$ \\
\hline Escala funcional & & & & \\
Desempenho físico & $81,4(21,9)$ & $64,7(34,9)$ & $-16,7$ & 0,008 \\
Desempenho funcional & $76,1(28,1)$ & $63,7(39,8)$ & $-12,4$ & 0,126 \\
Desempenho cognitivo & $85,1(21,4)$ & $82,7(24,2)$ & $-2,4$ & 0,717 \\
Desempenho emocional & $76,7(17,7)$ & $78,2(22,3)$ & $+1,5$ & 0,798 \\
Desempenho social & $77,4(28,7)$ & $73,2(38,0)$ & $-4,2$ & 0,595 \\
Escala de sintomas/itens únicos & & & & \\
Dor & $27,3(31,5)$ & $30,3(38,5)$ & $+3,0$ & 0,679 \\
Fadiga & $28,6(27,2)$ & $36,5(35,3)$ & $+7,9$ & 0,265 \\
Náuseas e vômitos & $14,3(21,1)$ & $14,9(22,4)$ & $+0,6$ & 0,912 \\
Dispneia & $17,9(32,0)$ & $21,4(36,5)$ & $+3,5$ & 0,698 \\
Insônia & $23,8(33,8)$ & $20,2(31,9)$ & $-3,6$ & 0,587 \\
Perda de apetite & $22,6(34,0)$ & $34,5(34,5)$ & $+11,9$ & 0,152 \\
Constipação & $21,4(35,4)$ & $14,3(32,0)$ & $-7,1$ & 0,441 \\
Diarreia & $7,1(22,9)$ & $14,3(35,6)$ & $+7,2$ & 0,339 \\
Dificuldades financeiras & $22,6(25,7)$ & $19,0(30,7)$ & $-3,6$ & 0,670 \\
Estado de saúde geral/QV & $69,3(23,1)$ & $64,3(32,2)$ & $-5,0$ & 0,413 \\
\hline
\end{tabular}

$\mathrm{dp}=$ desvio-padrão; $\mathrm{QV}=$ qualidade de vida; $\Delta(\mathrm{D}-\mathrm{A})=$ variação entre os escores no momento depois e antes do início do tratamento; *Teste $t$ Student. 
Quando avaliadas as médias dos escores do domínio "desempenho físico" por tipo de tumor, as pacientes com tumor de mama tinham média de 85,9 pontos e pioraram para uma média de 73,3 pontos no momento "depois" ( $\mathrm{p}=0,051)$. Para os pacientes com tumor de próstata, houve também uma piora das médias dos escores, sendo no momento "antes" de 70,7 pontos e 33,3 pontos no momento "depois" $(\mathrm{p}=0,118)$. Já para os pacientes com tumor de pulmão, as médias dos escores aumentaram, passando de 75,6 pontos para 78,9 pontos no momento "depois" ( $\mathrm{p}=0,734)$. Para o domínio "estado geral de saúde/QV" avaliado por tipo de neoplasia, houve diminuição da média dos escores para os pacientes com neoplasia da próstata, sendo 75,0 pontos antes do início da quimioterapia e 36,7 cerca de dois meses após $(\mathrm{p}=0,042)$, de acordo com a tabela 5 .

Tabela 5. Qualidade de vida no momento "antes" e "depois" do tratamento quimioterápico de acordo com o tipo da neoplasia de idosos atendidos no Instituto de Medicina Integral Prof. Fernando Figueira. Recife-PE, 2012-2013.

\begin{tabular}{lcccc}
\hline & $\begin{array}{c}\text { Antes } \\
\text { média (dp) }\end{array}$ & $\begin{array}{c}\text { Depois } \\
\text { média (dp) }\end{array}$ & $\begin{array}{c}\Delta \\
(\mathrm{D}-\mathrm{A})\end{array}$ & $\mathrm{p}^{*}$ \\
\hline Desempenho físico & & & & \\
$\quad$ Tumor de mama & $85,9(16,1)$ & $73,3(25,8)$ & $-12,6$ & 0,051 \\
Tumor de próstata & $70,7(31,5)$ & $33,3(41,1)$ & $-37,4$ & 0,118 \\
$\quad$ Tumor de pulmão & $75,6(23,4)$ & $78,9(25,8)$ & $+3,3$ & 0,734 \\
Estado de saúde geral/QV & & & & \\
Tumor de mama & $75,9(14,0)$ & $69,4(32,2)$ & $-6,5$ & 0,490 \\
Tumor de próstata & $75,0(16,7)$ & $36,7(30,4)$ & $-38,3$ & 0,042 \\
Tumor de pulmão & $63,9(24,0)$ & $81,9(14,4)$ & +18 & 0,115 \\
\hline
\end{tabular}

$\mathrm{dp}=$ desvio-padrão; $\mathrm{QV}=$ qualidade de vida; $\Delta(\mathrm{D}-\mathrm{A})=$ variação entre os escores no momento depois e antes do início do tratamento; *Teste t Student. 


\section{DISCUSSÃO}

Neste estudo, os pacientes pioraram o desempenho físico após o início da quimioterapia quando se compararam as médias dos escores do EORTC QLQ-C30 antes e cerca de dois meses após o início do tratamento. A escassez de estudos na literatura que avaliam a qualidade de vida de idosos em quimioterapia dificulta uma comparação direta com o presente estudo. ${ }^{17}$

A piora do domínio "desempenho físico", aqui encontrada, também já havia sido descrita por Doordjuin et al. ${ }^{9}$ em pacientes idosos pouco sintomáticos com linfoma não-Hodgkin agressivo, quando se avaliou o efeito da quimioterapia por doença avançada/sintomática. Observou-se piora no desempenho físico naqueles que eram pouco sintomáticos; já para aqueles com mais sintomas, o desempenho físico melhorou após o início da quimioterapia. Isto demonstra que o efeito do tratamento, no desempenho físico, talvez seja benéfico para aqueles que tenham muitos sintomas da doença antes de iniciar a quimioterapia.. Esse efeito pode ter ocorrido nos sete $(22,6 \%)$ pacientes portadores de neoplasia de pulmão que tiveram as médias de desempenho físico aumentadas, quando comparados os momentos "antes" e "depois" da quimioterapia., ${ }^{9,17}$

Quando se avaliam as médias dos escores do domínio "estado de saúde geral/QV" do EORTC QLQ-C30 nos momentos "antes" e "depois", evidencia-se que não houve modificação, exceto para a neoplasia da próstata, que piorou de maneira significativa. Tal achado pode ser explicado pela toxicidade da quimioterapia utilizada (docetaxel) em todos os pacientes. Dos cinco pacientes portadores de neoplasia de próstata, quatro tiverem a quimioterapia suspensa por toxicidade.

Um estudo ${ }^{12}$ que avaliou 1.006 pacientes portadores de neoplasia de próstata em quimioterapia demonstrou que, dos 669 que tinham realizado a mesma quimioterapia utilizada no presente estudo (docetaxel), 26\% apresentaram mais de um efeito adverso severo, estando a fadiga e diarreia entre os principais sintomas relatados. No entanto, mesmo com efeitos adversos significativos, houve melhora na QVRS. ${ }^{12}$ Numa análise retrospectiva do subgrupo de idosos deste estudo realizada em $2014,{ }^{17}$ ratificou-se que o docetaxel é tóxico para os idosos, e que tanto a tolerância quanto a eficácia dessa quimioterapia se reduzem com o avançar da idade, ainda persistindo o ganho na QVRS. O autor do referido artigo enfatiza que os idosos recrutados para o estudo eram mais saudáveis e tinham menos comorbidades do que a população em geral. Talvez isto justifique a não piora da QVRS da pesquisa supracitada, diferindo dos resultados aqui apresentados.

Outra explicação para os achados deste estudo pode estar associada ao fato de a avaliação do momento "depois" ter sido feita, em média, dois meses após o início da quimioterapia, quando os pacientes ainda estavam em tratamento. Nesse momento, os efeitos colaterais podem se sobrepor aos efeitos de melhora na doença, o que justificaria a piora evidenciada no desempenho físico. Não foi possível identificar se a piora do domínio "desempenho físico" aqui encontrada decorreu apenas da toxicidade do tratamento ou da evolução da neoplasia no intervalo de tempo entre a primeira e a segunda entrevista.

Resultados diversos sobre a modificação da QVRS de idosos em quimioterapia são descritos na literatura, enfatizando a heterogeneidade dessa população. Hurria et al. $^{18}$ propuseram uma estratificação de risco para idosos em quimioterapia que incorpora idade, tipo de quimioterapia, comorbidades, tipo de tumor, exames laboratoriais e variáveis da avaliação geriátrica ampla (AGA), mas seu estudo ainda carece de validação.

A forma mais recomendada para a decisão de um tratamento oncológico para pacientes idosos tem sido através da utilização da AGA, que apreende o idoso na multidimensionalidade com escalas confiáveis e já validadas de status funcional, desempenho físico, desempenho psicológico, comorbidades, condições socioeconômicas, síndromes geriátricas, polifarmácia, nutrição e apoio social. A AGA auxilia na avaliação da idade funcional do idoso, que em muitos pacientes difere da idade cronológica, e identifica 
sinais de envelhecimento acelerado que podem diminuir a tolerância do tratamento. ${ }^{19}$ Os estudos são encorajadores, mas a AGA ainda não foi incorporada na prática clínica, nem foi utilizada no presente estudo por ser demorada, exigir gastos e treinamento dos profissionais para sua aplicação..$^{17,19}$

Embora os pacientes do presente estudo não representem a população brasileira, por ter sido uma amostra de conveniência de um hospital terciário, as características clínicas encontradas foram muito semelhantes àquelas encontradas em idosos no Brasil. ${ }^{20}$ Mulheres são a maioria, cerca $70 \%$ da população de idosos ganham até dois salários mínimos e mais da metade tem HAS, concordando com os resultados do presente estudo. Chama atenção a maior quantidade de pacientes aposentados ou em licença saúde aqui relatados. O tratamento do câncer pode levar ao afastamento precoce das atividades de trabalho, contribuindo para o alto percentual de licença médica. ${ }^{21}$ Os tumores mais frequentes neste estudo também foram os mais relatados para a população, e o estadio avançado de doença também foi o mais encontrado, conforme relatado para idosos com câncer no Brasil. ${ }^{22}$

Os regimes de quimioterapia contendo antraciclina (como a ciclofosfamida) e taxanes (como o paclitaxel) são amplamente utilizados em tumores da mama; ${ }^{22}$ no presente estudo, todas as pacientes com essa neoplasia foram submetidas a antraciclicna e/ou taxane. A segunda combinação mais utilizada de quimioterapia foi a carboplatina associada ao paclitaxel, que é um regime ativo para várias neoplasias, ${ }^{22}$ dentre as quais a de pulmão, e que também foi utilizado na maioria dos pacientes com esse diagnóstico. Os pacientes com tumor de próstata utilizaram docetaxel, que é o quimioterápico frequentemente administrado em pacientes com este tipo de neoplasia. ${ }^{17}$

Como limitação do estudo, salienta-se o pequeno tamanho da amostra, que prejudica a generalização dos resultados descritos. O período de tempo entre a primeira e a segunda entrevista pode ter influenciado na piora do desempenho físico decorrente dos efeitos colaterais da quimioterapia, uma vez que cerca de
$20 \%$ dos pacientes interromperam o tratamento devido à toxicidade. Salientam-se, ainda, os vários tipos de neoplasias incluídos no estudo e os vários regimes de quimioterápicos aos quais os pacientes foram submetidos, o que dificulta a comparação das médias dos escores do estudo com pesquisas já publicadas.

O instrumento escolhido para avaliar a QVRS (EORTC QLQ -C30) foi validado para autoaplicação, o que não representa a realidade da população do presente estudo, cujo questionário foi aplicado por meio de entrevista, devido ao baixo grau de escolaridade. No entanto, é possível que esse fator não tenha contribuído de maneira negativa para os resultados aqui apresentados, visto que um estudo ${ }^{10}$ que utilizou o mesmo questionário entrevistou alguns pacientes por telefone e concluiu que a forma de administração do questionário não modifica as respostas.

É importante ressaltar que, mais recentemente, a EORTC criou um questionário específico para idosos, o EORTC QLQ-ELD14. Este aborda questões importantes para a QV do idoso, como independência funcional, relação com família e amigos, preocupações com o futuro, autonomia e quantidade de doença, mas só foi validado em agosto de 2013, quando o trabalho de campo do presente artigo já havia sido encerrado. ${ }^{23}$ Ademais, o local do estudo, por ser um hospital de referência em oncologia de uma população atendida pelo SUS, pode refletir as características dessa população e não representar os idosos de todos os estratos sociais.

\section{CONCLUSÃO}

O presente estudo observou que a quimioterapia antineoplásica piorou o desempenho físico dos idosos, mas não houve alteração da qualidade de vida desses pacientes, exceto para aqueles com câncer de próstata, que pioraram durante o tratamento.

Dessa forma, salienta-se a importância de continuar realizando mais estudos sobre qualidade de vida relacionada à saúde, para que se possa definir o tratamento mais adequado para essa população. 


\section{REFERÊNCIAS}

1. Thun MJ, DeLancey JO, Center MM, Jemal A, Ward EM. The global burden of cancer: priorities for prevention. Carcinogenesis 2010;31(1):100-10.

2. Cancer Research UK [Internet]. London: Cancer Research UK; [2002?]. Cancer Mortality by age. 20102012 [acesso em 08 de. 2014]; [aproximadamene 2 telas]. Disponível em http://www.cancerresearchuk. org/cancer-info/cancerstats/mortality/age/uk-cancermortality-statistics-by-age\#cancer.

3. DeSantis CE, Chun Chieh L, Mariotto AB, Siegel RL, Stein $\mathrm{KD}$, et al. Cancer treatment and survivorship statistics, 2014 CA Cancer J Clin 2014;64(4):252-71.

4. Freire MEM, Sawada MO, De França ISX, Da Costa SFG, Oliveira CDB. Qualidade de vida relacionada à saúde de pacientes com câncer avançado: uma revisão integrativa. Rev Esc Enferm USP 2014;48(2):357-67.

5. Wildiers H, Heeren P, Puts M, Topinkova E, JanssenHeijnen ML, Extermann M, et al. Internacional Society of Geriatric Oncology Consensus on Geriatirc Assessment in Older Patients With Cancer. J Clin Oncol 2014;32(24):2595-603.

6. Aaroson NK, Ahmedzai S, Bergman B, Bullinger M, Cull A, Duez N, et al. The European Organization for research and treatment of Cancer QLC-C30: a quality-of-life instrument for use in international clinical trials in oncology. J Natl Cancer Inst 1993;85(5):365-76.

7. Hilpert F, Du Bois A, Greimel ER, Hedderich J, Krause G, Venhoff L, et al. Feasibility, toxicity and quality of life of first-line chemotherapy with platinum/paclitaxel in elderly patients aged $\geq 70$ years with advanced ovarian cancer: a study by the AGO OVAR Germany. Ann Oncol 2007;18(2):282-87.

8. Biesma B, Wymenga AN, Vincent A, Dalesio O, Smit HJ, Stigt JA, et al. Quality of life, geriatric assessment and survival in elderly patients with non-small-cell lung cancer treated with carboplatin-gemcitabine or carboplatin-paclitaxel: NVALT-3 a phase III study. Ann Oncol 2011;22(7):1520-27.

9. Doorduijn J, Buijt I, Holt B, Steijaert M, Uyl-de Groot C, Sonneveld P. Self-reported quality of life in elderly patients with aggressive Non-Hodgkin's lymphoma treated with CHOP chemotherapy. Eur J Haematol 2005;75(2):116-23.
10. Browall MM, Ahlberg KM, Persson LO, Karlsson PO, Danielson EB. The impact of age on healthrelated quality of life (HRQol) and symptoms among postmenopausal women with breast cancer receiving adjuvant chemotherapy. Acta Oncol 2008;47(2):207-15.

11. Gridelli C. The ELVIS Trial: a phase III study of a single-agent Vinorelbine as first- line treatment in elderly patients with advanced non-small cell lung cancer. Oncologist 2001;6 Suppl 1:4-7.

12. Berthold DR, Pond GR, Soban F, De Wit R, Eisenberger M, Tannock IF. Docetaxel plus prednisone or Mitoxantrone plus prednisone for advanced prostate cancer: uptodated survival in TAX 327. J Clin Oncol 2008;26(2):242-5.

13. Owusu C, Koroukian SM, Schluchter M, Bakaki P, Berger NA. Screnning older cancer patients for a Comprehensive Geriatric Assessment: a comparison of three instruments. J geriatr Oncol 2011;2(2):121-9.

14. Franceschini J, Jardim JR, Fernandes ALG, Jamnik S, Santoro IL. Reprodutibilidade da versão em português do Brasil do European Organization for Research and Treatment of Cancer Core Quality of Life Questionnaire em conjunto com seu módulo específico para câncer de pulmão. J Bras Pneumol [Internet] 2010 [acesso em 08 dez 2014];36(5):595-602. Disponível em: http://www. scielo.br/scielo.php?script=sci_arttext\&pid=S180637132010000500011\&lng=en.

15. Brasil. Portaria n ${ }^{\circ} 102$, de 03 de Fevereiro de 2012. Dispõe sobre Centro de Assistência de Alta Complexidade em Oncologia (CACON). Saúde Legis. 2012. Disponível em: http://bvsms.saude.gov.br/bvs/ saudelegis/sas/2012/prt0102_03_02_2012.html

16. Schaafsma J, Osoba D. The Karnofsky performance status scale re-examined: a cross validation with the EORTC-C30. Qual life Res 1994;3(6):413-24.

17. Horgan AM, Seruga B, Pond GR, Alibhai SM, Amir E, De Wit R, et al. Tolerability and efficacy of docetaxel in older men with metastatic castrateresistant prostate cancer (mCRPC) in the TAX 327 trial. J Geriatr Oncol 2014;5(2):119-26.

18. Hurria A, Togawa K, Mohile SG, Owusu C, Klepin HD, Gross CP, et al . Predicting chemotherapy toxicity in older adult with cancer: a prospective multicenter study. J Clin Oncol 2011;29(25):3457-65. 
19. Davidoff AJ, Tang M, Seal B, Edelman MJ. Chemotherapy and survival benefit in elderly patients with advanced non-small-cell lung cancer. J Clin Oncol. J Clin Oncol 2010;28(13):2191-97.

20. Instituto Brasileiro de Geografia e Estatística. Indicadores Sociodemográficos e de Saúde no Brasil [Internet]. Rio de Janeiro: IBGE; 2009 [acesso em 3 jan 2014]. 152 p. Disponível em: http://www. ibge.gov.br/home/estatistica/populacao/indic_ sociosaude/2009/indicsaude.pdf.
21. Mansano-Schlosser TC, Ceolim MF. Fatores associados à qualidade do sono de idosos submetidos à quimioterapia. Rev Latinoam Enferm 2012;20(6):1100-8.

22. Instituto Nacional do Câncer [Internet]. Rio de Janeiro: INCA. 1996- .; [acesso em 26 jan 2012]; Disponível em: http://www.inca.gov.br/estimativa/2012.

23. Wheelwright S, Darlington AS, Fitzsimmons D, Fayers P, Arraras JI, Bonnetain F, et al. International validation of the EORTC QLQ-ELD14 questionnaire for assessment of health-related quality of life elderly patients with cancer. Br J Cancer 2013;109(4):852-58.

Recebido: $12 / 2 / 2014$

Revisado: $13 / 10 / 2014$

Aprovado: 09/12/2014 